\title{
Intraperitoneal administration of paclitaxel and oral S-1 for a patient with peritoneal dissemination and hydronephrosis due to advanced gastric cancer
}

\author{
Shigeyuki Tamura, Hirofumi Miki, Ken Nakata, Daisuke Takiuchi, Kaoru Okada, Shin Nakahira, \\ Shu Okamura, Keishi Sugimoto, Naohiro Tomita, and Yuichi Takatsuka
}

Department of Surgery, Kansai Rosai Hospital, 3-1-69 Inabaso, Amagasaki 660-8511, Japan

\begin{abstract}
We report a patient with type 3 gastric cancer with peritoneal dissemination and hydronephrosis who was successfully treated with intraperitoneal infusion of paclitaxel and oral administration of S-1. He was diagnosed with unresectable gastric cancer with severe peritoneal dissemination by staging laparoscopy. We selected combined chemotherapy with both paclitaxel and S-1. Paclitaxel at $60 \mathrm{mg} / \mathrm{m}^{2}$ was administered intraperitoneally on days 1 and 8 , and $S-1$ at $100 \mathrm{mg} / \mathrm{body}$ was administered orally for 14 days, followed by 7 days' rest, as one course. After five courses, primary tumor reduction was confirmed and no cancer cells were detected on pathocytological investigation at second-look laparoscopy. The patient underwent total gastrectomy with lymph node dissection. He died from liver metastasis 29 months after the initial treatment, but he had not suffered from peritoneal metastases and had kept a good quality of life (QOL) since that treatment. This chemotherapy can be applied as one of the promising candidates for the treatment of patients with peritoneal metastasis of gastric cancer.
\end{abstract}

Key words Advanced gastric cancer - Peritoneal dissemination · Intraperitoneal chemotherapy $\cdot$ Paclitaxel $\cdot$ S-1

\section{Introduction}

The prognosis of gastric cancer patients with peritoneal dissemination is extremely poor [1]. For that reason, various clinical attempts have been made to treat the peritoneal dissemination of gastric cancer, including systemic chemotherapy [2], intraperitoneal chemotherapy and/or hyperthermia [3], and aggressive surgery [4]. However, the results of these therapies have been unsatisfactory.

Offprint requests to: $\mathrm{S}$. Tamura

Received: February 23, 2007 / Accepted: June 25, 2007
S-1 has been developed in Japan as an oral anticancer drug, composed of tegafur, 5-chloro-2, 4-dihydroxypyridine (gimeracil), and monopotassium 1, 2, 3, 4tetrahydro-2, 4-dioxo-1, 3, 5-triazine-6-carboxylate (oteracil), based on the biological modulation of 5fluorouracil (FU) [5]. Japanese late phase II trials of S-1 in gastric cancer have shown overall response rates of up to $49 \%$ and a median survival period of 8 months $[6,7]$. We have demonstrated that S-1 has a larger area under the curve 5-FU for peritoneal dissemination and ascites than for plasma [8], and thus S-1 might be effective for prolonging the survival of gastric cancer patients with peritoneal metastasis.

Paclitaxel is a cytotoxic antineoplastic agent that results in tumor cell death by causing the excessive polymerization of tubulin and microtubule dysfunction [9]. The large molecular weight and bulky chemical structure of paclitaxel delay its peritoneal clearance [10] and increase exposure in the peritoneal cavity, and thus it can be exploited in the treatment of gastric cancer with peritoneal dissemination.

We report a gastric cancer patient with peritoneal dissemination and hydronephrosis who was successfully treated with intraperitoneal infusion of paclitaxel and oral administration of S-1.

\section{Case report}

A 34-year-old man was treated with an H2-blocker antagonist for about 6 months for a chief complaint of upper abdominal discomfort, suggesting the possibility of duodenal ulcer. Because his condition did not improve, he underwent endoscopic examination at another clinic. He was diagnosed as having type 3 advanced gastric cancer on the greater curvature of the gastric body by upper gastrointestinal (UGI) and endoscopic examination. He was referred to a hospital for surgical resection. An abdominal computed tomogra- 

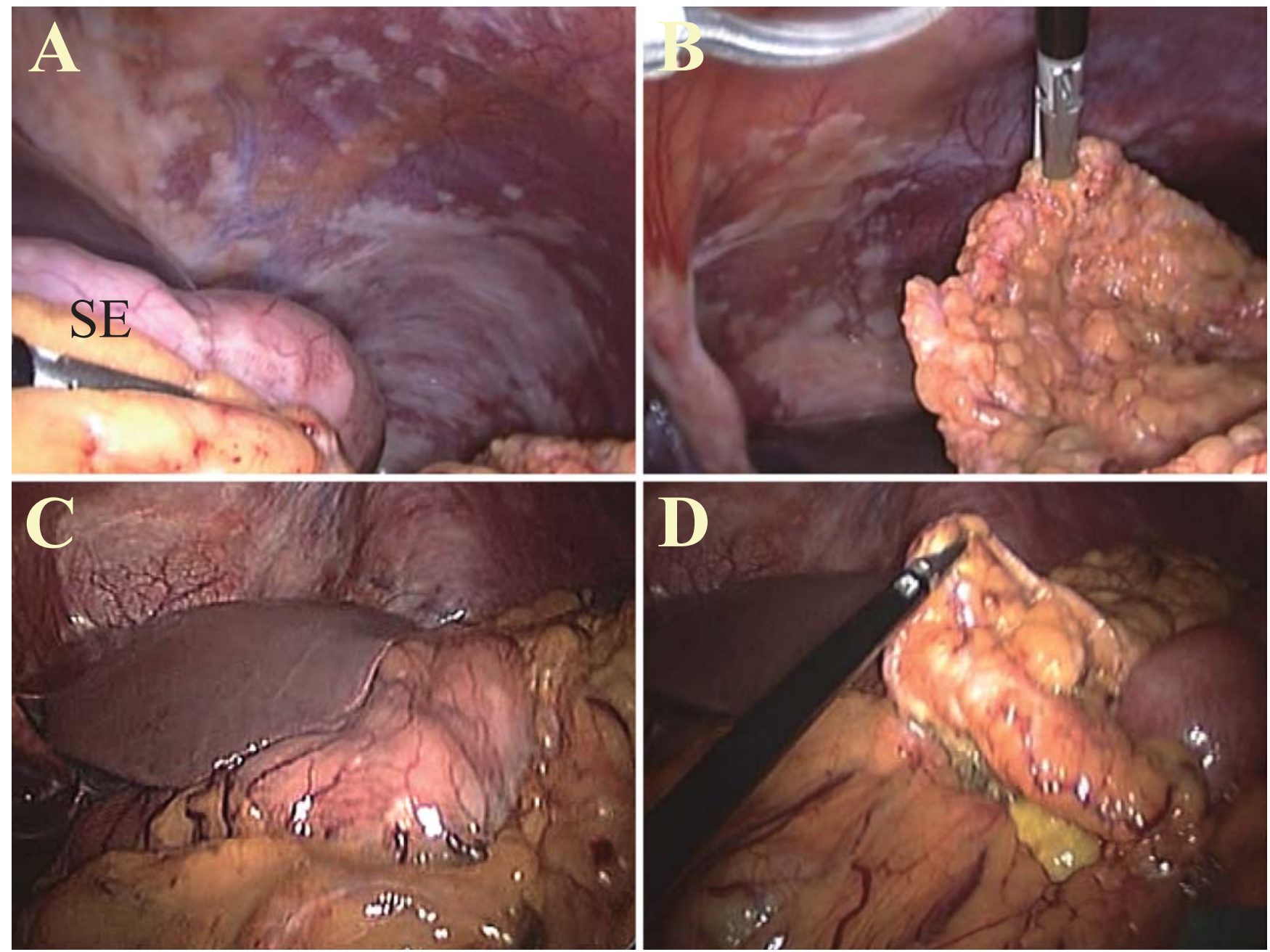

Fig. 1A-D. Laparoscopic examination findings: A, B before and C, D after five courses of intraperitoneal chemotherapy. C, D Lesions of peritoneal dissemination were found to have changed to normal peritoneum or small white nodules, and no adenocarcinoma cells were found histologically in nodes of the omentum or in lavage fluid. $S E$, serosal exposure

phy (CT) scan showed a small amount of ascites, and hydronephrosis of the right kidney, suggesting peritoneal metastasis. The carcinoma was judged to be unresectable and he was referred to our hospital. Staging laparoscopy was performed in October 2002; ascites was collected and biopsy specimens were taken from the greater omentum, showing multiple nodules (Fig. 1A,B). Pathocytological investigation of biopsy specimens and ascites showed poorly differentiated adenocarcinoma, diagnosed as P1, CY1, and stage IV according to the Japanese classification of gastric carcinoma [11].

\section{Treatment schedules}

After a Double-J catheter was intubated for the hydronephrosis of the right kidney, combination chemotherapy was started. Paclitaxel was administered intraperitoneally in $500 \mathrm{ml}$ normal saline as rapidly as possible following premedication, using intraperitoneal catheters attached to a subcutaneous portal delivery system, which was placed at the staging laparoscopy. Paclitaxel at $60 \mathrm{mg} / \mathrm{m}^{2}$ was administered on days 1 and 8 , and S-1 at $100 \mathrm{mg} /$ body was administered orally for 14 days, followed by 7 days' rest, as one course. This treatment cycle was repeated five times, and the patient's response was evaluated. In advance of this therapy, informed consent was obtained from the patient and his family.

\section{Objective response and toxicities}

The response was evaluated after five cycles, by UGI, gastrointestinal endoscopy, and CT. UGI and endoscopy showed remarkable tumor regression. CT after chemotherapy showed a remarkable reduction in the thickness of the gastric wall and the disappearance of ascites. As the objective response after five courses of 
chemotherapy was evaluated as a partial response, second-look laparoscopy was performed to evaluate the effects of chemotherapy on the peritoneal lesions. Lesions of peritoneal dissemination were found to have changed to white nodules, and no adenocarcinoma cells were found pathocytologically in the nodes of the omentum or in lavage fluid (Fig. 1C,D).

No gastrointestinal or bone marrow toxicities were recognized in the chemotherapy courses, except for a sole adverse effect on serum total bilirubin level (peak level of bilirubin, $3.5 \mathrm{mg} / \mathrm{dl}$ ).

\section{Operative findings}

A gastric tumor was recognized on the greater curvature of the gastric body, showing invasion to the serosa. Although multiple white scars were recognized in the omentum and peritoneum, no tumor cells were observed histologically in frozen sections of the peritoneum and omentum. Also, peritoneal lavage cytology was negative, and we performed total gastrectomy with D2 lymph node dissection without splenectomy. Macroscopic findings of the resected specimen showed an irregular depressed lesion with fibrosis through the wall of the stomach (Fig. 2). Microscopically, the resected specimens showed poorly differentiated adenocarcinoma which had invaded to the subserosa, while most parts of the tumor showed severe fibrosis (Fig. 3), judged to be a grade 2 effect following chemotherapy. Because small numbers of cancer cells were detected in the largest nodule of the omentum, the final findings showed that the tumor was T2, N1 (\#3), H0, P1, CY0, and stage IV according to the Japanese classification of gastric carcinoma.

\section{Postoperative course}

The patient did well after the operation. S- 1 was administered orally as adjuvant chemotherapy, at a dose of $100 \mathrm{mg} /$ day twice daily for 14 days, followed by a 1 -week rest period. A solitary liver metastasis was recognized by abdominal CT 3 months after the operation. Intravenous administration of paclitaxel was added biweekly at the dose of $90 \mathrm{mg} /$ body.

CT showed a complete response (CR) of the liver metastasis at 5 months after the beginning of this intravenous paclitaxel administration. Twelve months after the CR of liver metastasis, the patient maintained a good quality of life and the performance status remained at grade 0 , according to the WHO grading, without any signs of peritoneal dissemination. However, the liver metastasis was found to be re-growing 21 months after the operation, and he died from liver metastasis 29 months after the initial chemotherapy.

\section{Discussion}

The most common cause of death in gastric cancer patients with noncurative resection and recurrence is peritoneal dissemination. Although patients with this disease have been treated after surgical resection with high-dose systemic or intraperitoneal chemotherapy, the results of these treatments have been poor, and most patients with peritoneal dissemination die within 6 months after diagnosis [1].

For patients with peritoneal dissemination, systemic chemotherapy with methotrexate (MTX)/5-FU or intraoperative chemohyperthermia has been performed. Konishi et al. [12] reported that an MTX/5FU regimen showed higher efficacy in patients with diffuse type of adenocarcinoma than in those with intestinal type. The response rate of patients with peritoneal dissemination in their study was $23 \%(6 / 26)$ and ascites was eliminated in 8 of 16 patients $(50 \%)$. Tahara et al. [13] reported that sequential MTX/5FU therapy was effective for patients with confirmed peritoneal dissemination, and objective improvement of ascites was seen in 13 of 26 patients, including 5 who showed complete disappearance of the ascites. Although the median survival time and median time to treatment failure were 259 days and 167 days, respectively, the results of this therapy have been unsatisfactory.

$\mathrm{S}-1$ is an oral fluoropyrimidine anticancer drug that combines three pharmacological agents: tegafur, gimeracil, and oteracil potassium [5]. Late phase II studies of S-1 in advanced gastric cancer obtained overall response rate of $46 \%$ and $49 \%$ [6, 7]. Also, several reports have demonstrated that S-1 was effective for the undifferentiated type of histology (such as poorly differentiated adenocarcinoma and signet-ring cell carcinoma), which is relevant to peritoneal dissemination [14]. Animal studies have revealed that the concentration of 5-FU in intraperitoneal fluid remained high after the oral administration of S-1. And we have demonstrated that S-1 was effective in preventing the development of peritoneal dissemination [8].

Although S-1 may be a promising agent for peritoneal dissemination due to gastric cancer, the effectiveness of S-1 for peritoneal dissemination is not clear, because the two aforementioned late phase II studies excluded patients with nonmeasurable metastatic lesions such as peritoneal dissemination [6, 7]. In our patient with severe peritoneal dissemination, we searched for a combined chemotherapy in the hope of achieving a more effective response in a short time.

Paclitaxel has a high molecular weight and bulky structure, delaying its clearance from the peritoneal cavity; a profound intraperitoneal exposure advantage for paclitaxel was demonstrated with intraperitoneal/ systemic AUC ratios ranging from 336 to 2890 (mean + 


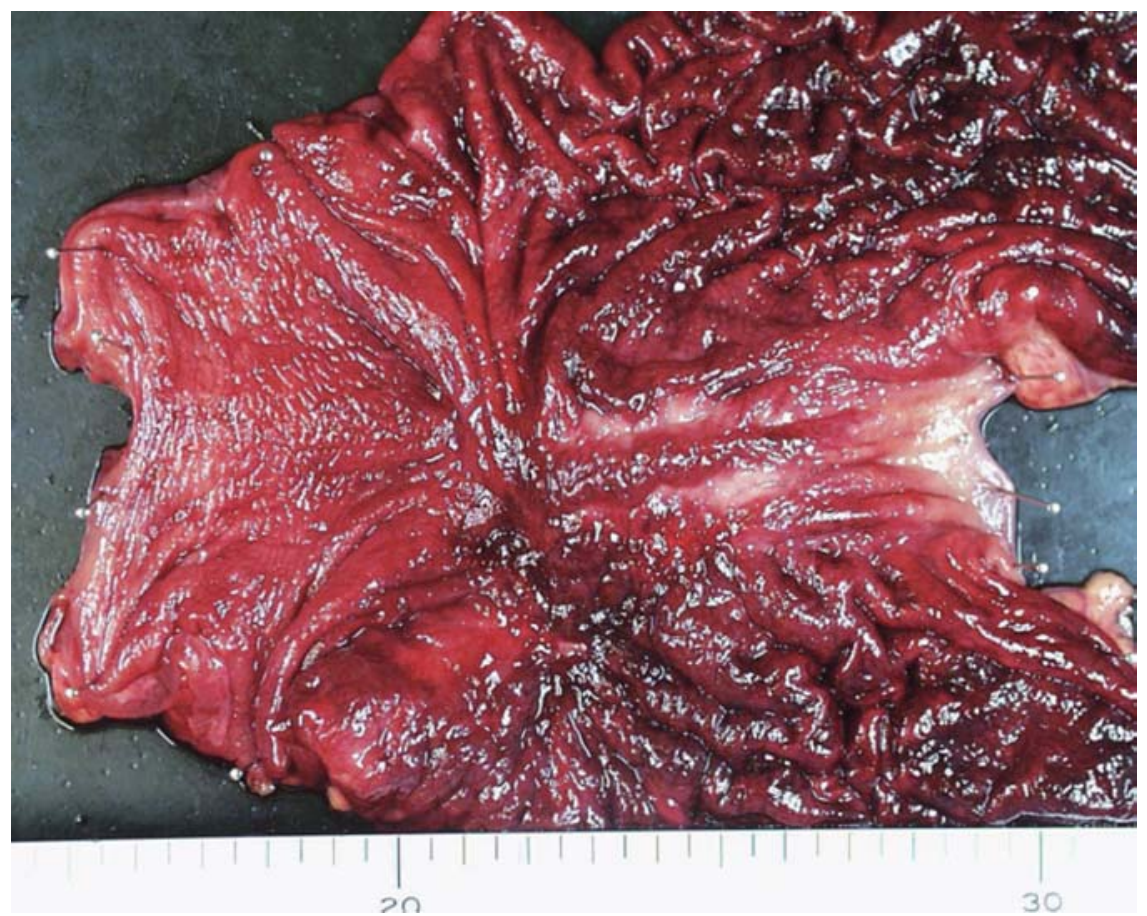

Fig. 2. Macroscopic findings of the resected specimen showed an irregular depressed lesion with fibrosis through the wall of the stomach

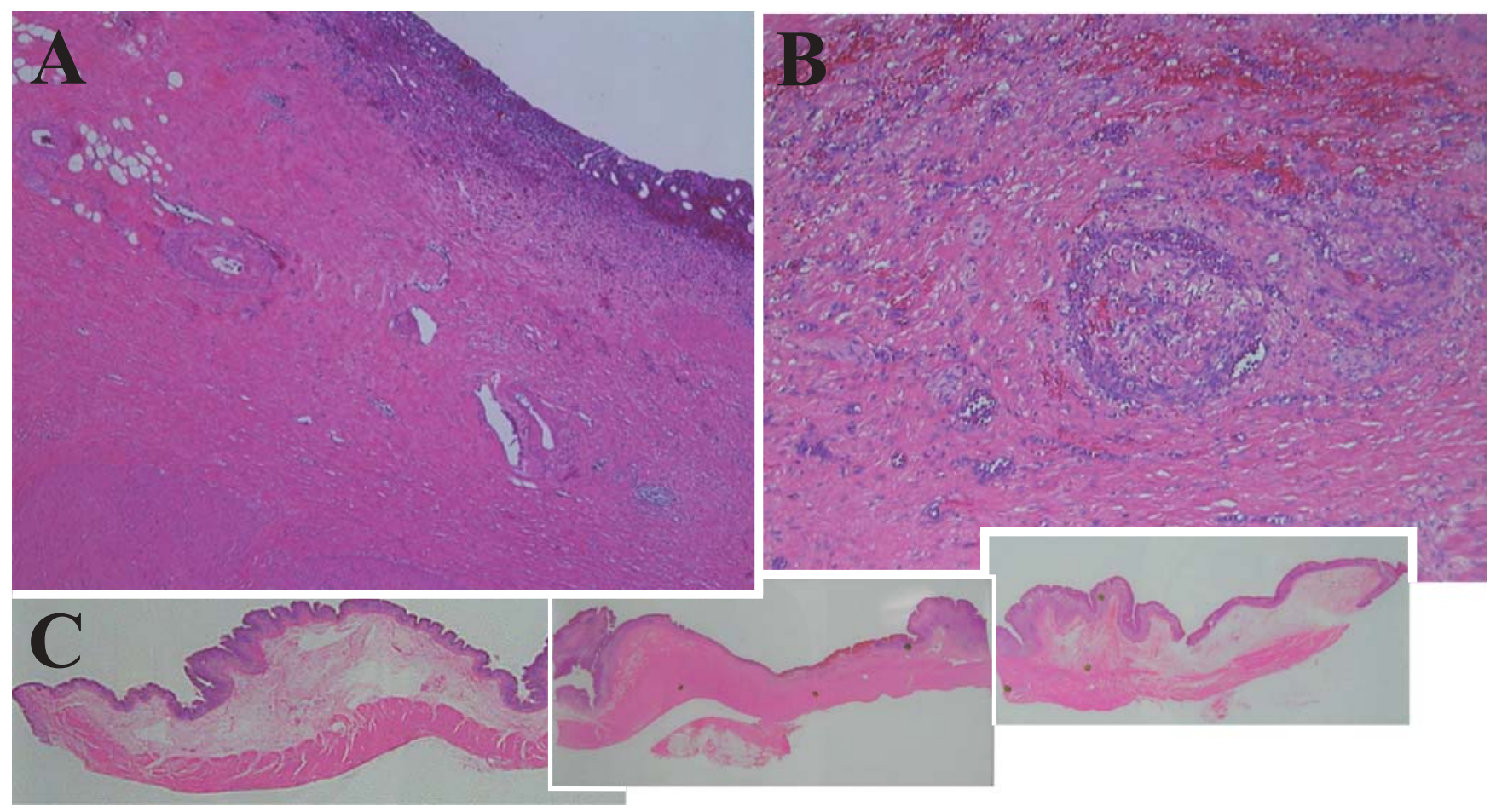

Fig. 3A-C. Microscopic findings of the excised specimen. The resected specimen showed poorly differentiated adenocarcinoma which had invaded to the subserosa, while most parts of the tumor from the submucosa to the subserosa showed change to severe fibrosis. A H\&E, $\times 40 ;$ B H\&E, $\times 100 ;$ C H\&E, $\times 2$ 
SE, $996+93$ ) [10]. Concerning the dosing schedule of weekly intraperitoneal paclitaxel, Francis et al. [15] have demonstrated that doses up to $60 \mathrm{mg} / \mathrm{m}^{2}$ per week were well tolerated, with minimal abdominal pain or systemic toxicity with potentially cytotoxic concentrations of paclitaxel of 5 to 7 days' duration. In addition, in a phase II trial of intraperitoneal paclitaxel in carcinoma of the ovary, tube, and peritoneum, a weekly dosing schedule of intraperitoneal paclitaxel, for a total of 16 weeks, was tolerable and active in patients with microscopic residual disease [16]. In that study, although the patients with microscopic disease achieved a good response, patients with any macroscopic disease achieved only a limited effect. Therefore, we adopted a combination of intraperitoneal administration of paclitaxel and oral S-1 for the present patient with high peritoneal dissemination of gastric cancer in the hope of an effect on both microscopic and macroscopic diseases. Although several therapeutic methods have been reported concerning the intraperitoneal administration of taxanes in patients with gastric cancer [17, 18], there is no established schedule for their use in patients with peritoneal dissemination.

We confirmed the dramatic effect of our therapy by laparoscopic examination and we carried out ablative surgery and achieved a survival time of more than 2 years without any adverse effects. This chemotherapy can be applied as one of the promising candidates for the treatment of patients with peritoneal metastasis of gastric cancer. To obtain clear evidence about first-line chemotherapy for patients with peritoneal dissemination, a randomized clinical trial, comparing S-1 alone and S-1 plus other anticancer agents such as taxanes, will be necessary. In Japan, there are several problems concerning expansion of the clinical utilization of intraperitoneal administration of paclitaxel; thus, a phase I/II clinical trial for establishing the optimal dose and administration schedule of this drug is warranted in the near future.

\section{References}

1. Yonemura Y, Sawa T, Kinoshita K, Matsuki N, Fushida S, Tanaka $\mathrm{S}$, et al. Neoadjuvant chemotherapy for high-grade advanced gastric cancer. World J Surg 1993;17:256-62.

2. Sugerbaker PH, Yonemura Y. Clinical pathway for the management of resectable gastric cancer with peritoneal seeding: best palliation with a ray of hope for cure. Oncology 2000;58:96107.

3. Fujimoto S, Takahashi M, Kobayashi K, Kure M, Mutou T, Masaoka $\mathrm{H}$, et al. Relation between clinical and histologic outcome of intraperitoneal hyperthermic perfusion for patients with gastric cancer and peritoneal metastasis. Oncology 1993;50: 338-43.

4. Nomura E, Niki M, Fujii K, Shinohara H, Nishiguchi K, Sonoda T. Efficacy of intraperitoneal and intravenous chemotherapy and left upper abdominal evisceration for advanced gastric cancer. Gastric Cancer 2001;4:75-82.

5. Shirasaka T, Shimamoto Y, Ohshimo H, Yamaguchi M, Kato T, Yonekura K, et al. Development of a novel form of an oral 5fluorouracil derivative (S-1) directed to the potentiation of the tumor selective cytotoxicity of 5-fluorouracil by two biochemical modulators. Anticancer Drugs 1996;7:548-57.

6. Sakata Y, Ohtsu A, Horikoshi N, Sugimachi K, Mitachi Y, Taguchi T. Late phase II study on novel oral fluoropyrimidine anticancer drug S-1 (IM Tegafur-0.4M Gimestat-1M Otastat Potassium) in advanced gastric cancer patients. Eur J Cancer 1998;34:1715-20.

7. Koizumi W, Kurihara M, Nakano S, Hasegawa K. Phase II study of S-1, a novel oral derivative of 5-fluorouracil, in advanced gastric cancer. Oncology 2000;58:191-7.

8. Mori T, Fujiwara Y, Yano M, Tamura S, Yasuda T, Takiguchi S, et al. Prevention of peritoneal metastasis of human gastric cancer cells in nude mice by S-1, a novel oral derivative of 5-fluorouracil. Oncology 2003;64:176-82.

9. Rowinsky EK, Donehower RC, Jones RJ, Tucker RW. Microtubule changes and cytotoxicity in leukemic cell lines treated with Taxol. Cancer Res 1988;48:4093-100.

10. Markman M, Rowinsky E, Harkes T, Reichman B, Jones W, Lewis JL Jr, et al. Phase I trial of intraperitoneal Taxol: a Gynecoloic Oncology Group Study. J Clin Oncol 1992;10:1485-91.

11. Japanese Gastric Cancer Association. Japanese classification of gastric carcinoma. 2nd English ed. Gastric Cancer 1998;1:25-30.

12. Konishi T, Hiraishi M, Mafune K, Miyama T, Hirata T, Mori K, et al. Therapeutic efficacy and toxicity of sequential methotrexate and 5-fluorouracil in gastric cancer. Anticancer Res 1994: 14: 1277-80.

13. Tahara M, Ohotsu A, Boku N, Nagashima F, Muto M, Sano Y, et al. Sequential methotrexate and 5-fluorouracil therapy for gastric cancer patients with peritoneal dissemination: a retrospective study. Gastric Cancer 2001;4:212-8.

14. Osugi H, Takada N, Takemura M, Kaseno S, Lee S, Ueno M, et al. Oral fluoropyrimidine anticancer drug TS-1 for gastric cancer patients with peritoneal dissemination. Oncol Rep 2002;9:811-5.

15. Francis P, Rowinsky E, Schneider J, Hakes T, Hoskins W, Markman M. Phase I feasibility and pharmacologic study of weekly intraperitoneal paclitaxel: a Gynecologic Oncology Group pilot study. J Clin Oncol 1995;13:2961-7.

16. Markman M, Brady MF, Spirtos NM, Hanjani P, Rubin SC. Phase II trial of intraperitoneal Taxol in carcinoma of the ovary, tube, and peritoneum: a Gynecoloic Oncology Group Study. J Clin Oncol 1998;16: 1485-91.

17. Fushida S, Furui N, Kinami S, Ninomiya I, Fujimura T, Nishimura G, et al. Pharmacologic study of intraperitoneal paclitaxel in gastric cancer patients with peritoneal dissemination (in Japanese). Gan To Kagaku Ryoho (Jpn J Cancer Chemother) 2002;29:2164-7.

18. Yonemura Y, Endou Y, Bando E, Kuno K, Kawamura T, Kimura $\mathrm{M}$, et al. Effect of intraperitoneal administration of docetaxel on peritoneal dissemination of gastric cancer. Cancer Lett 2004;210: 189-96. 